\title{
A validation of technologies monitoring dairy cow feeding, ruminating, and lying behaviors
}

\author{
M. R. Borchers, ${ }^{*}$ Y. M. Chang, $†$ I. C. Tsai, ${ }^{*}$ B. A. Wadsworth, ${ }^{*}$ and J. M. Bewley* \\ *Department of Animal and Food Sciences, University of Kentucky, Lexington 40546 \\ †Research Support Office, Royal Veterinary College, University of London, London, United Kingdom NW1 0TU
}

\begin{abstract}
The objective of this study was to evaluate commercially available precision dairy technologies against direct visual observations of feeding, rumination, and lying behaviors. Primiparous $(\mathrm{n}=24)$ and multiparous $(\mathrm{n}=24)$ lactating Holstein dairy cattle (mean \pm standard deviation; $223.4 \pm 117.8 \mathrm{~d}$ in milk, producing 29.2 $\pm 8.2 \mathrm{~kg}$ of milk/d) were fitted with 6 different triaxial accelerometer technologies evaluating cow behaviors at or before freshening. The AfiAct Pedometer Plus (Afimilk, Kibbutz Afikim, Israel) was used to monitor lying time. The CowManager SensOor (Agis, Harmelen, Netherlands) monitored rumination and feeding time. The HOBO Data Logger (HOBO Pendant G Acceleration Data Logger, Onset Computer Corp., Pocasset, MA) monitored lying time. The CowAlert IceQube (IceRobotics Ltd., Edinburgh, Scotland) monitored lying time. The Smartbow (Smartbow GmbH, Jutogasse, Austria) monitored rumination time. The Track A Cow (ENGS, Rosh Pina, Israel) monitored lying time and time spent around feeding areas for the calculation of feeding time. Over 8 d, 6 cows per day were visually observed for feeding, rumination, and lying behaviors for $2 \mathrm{~h}$ after morning and evening milking. The time of day was recorded when each behavior began and ended. These times were used to generate the length of time behaviors were visually observed. Pearson correlations (r; calculated using the CORR procedure of SAS Version 9.3, SAS Institute Inc., Cary, NC), and concordance correlations (CCC; calculated using the epiR package of $\mathrm{R}$ version 3.1.0, $\mathrm{R}$ Foundation for Statistical Computing, Vienna, Austria) evaluated association between visual observations and technology-recorded behaviors. Visually recorded feeding behaviors were moderately correlated with the CowManager SensOor $(\mathrm{r}=0.88, \mathrm{CCC}=0.82)$ and Track A Cow $(\mathrm{r}=0.93$, $\mathrm{CCC}=0.79)$ monitors. Visually recorded rumination
\end{abstract}

Received December 31, 2015.

Accepted May 20, 2016.

${ }^{1}$ Corresponding author: jbewley@uky.edu behaviors were strongly correlated with the Smartbow $(\mathrm{r}=0.97, \mathrm{CCC}=0.96)$, and weakly correlated with the CowManager SensOor $(r=0.69, \mathrm{CCC}=0.59)$. Visually recorded lying behaviors were strongly correlated with the AfiAct Pedometer Plus ( $\mathrm{r}>0.99$, CCC >0.99), CowAlert IceQube $(\mathrm{r}>0.99$, CCC $>0.99)$, and Track A Cow $(r>0.99, \mathrm{CCC}>0.99)$. The HOBO Data Loggers were moderately correlated $(\mathrm{r}>0.83, \mathrm{CCC}>0.81)$ with visual observations. Based on these results, the evaluated precision dairy monitoring technologies accurately monitored dairy cattle behavior.

Key words: precision dairy monitoring, feeding behavior, ruminating behavior, lying behavior

\section{INTRODUCTION}

Many precision dairy-monitoring technologies claim to monitor udder health, estrus events, feet and leg health, and metabolic health (Rutten et al., 2013). These technologies provide benefit to producers and researchers by frequently monitoring dairy cattle without disturbing natural behavioral expression (Müller and Schrader, 2003). Dairy producers purchase precision dairy-monitoring technologies to improve individual animal, pen, and whole-farm management, increasing overall farm production efficiency (Wathes et al., 2008). However, for precision dairy-monitoring technologies to increase labor and production efficiency, they must easily and accurately quantify meaningful physiological or behavioral parameters (Senger, 1994).

The automatic measurement of chewing and ruminating activity can enable the early detection of feeding deficiencies and assist in ration adjustments (Zehner et al., 2012). Feeding and ruminating behaviors have traditionally been monitored through visual observation or video recording methods (Schirmann et al., 2009), but these methods are time consuming and only practically used in research settings. Additionally, monitoring animal behaviors using visual observation is subjective and open to observer interpretation (Weary et al., 2009). Monitoring rumination and feeding behaviors with precision dairy-monitoring technologies could replace 
subjective visual observations while providing useful and continuous measures of these behaviors. Technologies recording feeding and rumination behaviors have traditionally quantified these behaviors using chewing activity monitors (pressure and strain recorders; Beauchemin et al., 1989; Kononoff et al., 2002; Zehner et al., 2012). Chewing activity (pressure and strain) and feeding behavior monitors are primarily used in research settings, but commercially available rumination and feeding behavior quantification methods have recently been developed and evaluated. Bikker et al. (2014) evaluated a technology (CowManager Sensoor, Agis, Harmelen, the Netherlands) monitoring rumination and feeding behavior through head movement and found these behaviors to be closely related to visual observations. Similarly, Schirmann et al. (2009) evaluated a technology (HR Tag, SCR Engineers Ltd., Netanya, Israel) quantifying rumination sounds through a microphone and microprocessor and found a strong correlation between visual observations and technology. Another method to quantify feeding behaviors is through technologies describing when cows approach feeding areas. Many of these types of technologies have been evaluated and found to be highly correlated with visual methods (DeVries et al., 2003; Chapinal et al., 2007). These findings indicate the potential for technology performance to vary among technologies monitoring the same parameters through different methods.

Lying behavior is a parameter frequently quantified by precision dairy monitoring technologies (McGowan et al., 2007; O'Driscoll et al., 2008; Ledgerwood et al., 2010). Time spent lying can indicate cow comfort, welfare, and health changes (Haley et al., 2000). Proudfoot et al. (2014) found sick or ill cattle spent more time lying apart from the herd. Compared with other parameters measured by precision dairy-monitoring technologies (e.g., feeding behavior, rumination, and activity), standing and lying events are easily visually monitored but the process remains time-consuming.

Previous studies evaluating lying behavior have reported strong correlations between technologies and visual or video monitoring. Lying behaviors measured by the HOBO Data Logger (HOBO Pendant G Acceleration Data Logger, Onset Computer Corporation, Pocasset, MA) have strongly matched video monitoring observations (Ledgerwood et al., 2010). Similarly, the AfiAct Pedometer Plus (Afimilk, S.A.E. Afikim, Kibbutz Afikim, Israel) and IceTag (a version of the IceQube intended for research; IceRobotics Ltd., Edinburgh, Scotland) monitored dairy cow lying behavior and observations were closely related to video monitoring observations (Mattachini et al., 2013a,b).

Behavioral recording methods have rarely been compared on the same animals over the same periods. The objective of the current study was to evaluate commercially available precision dairy monitoring technologies against direct visual observations for feeding, rumination, and lying behaviors. To our knowledge, this study will also serve as the first to validate the Track A Cow (ENGS, Rosh Pina, Israel) system for feeding and lying behaviors, and the Smartbow (Smartbow GmbH, Jutogasse, Austria) system for rumination behavior. The hypothesis of our study is that lying behaviors will most closely match visual observations. Ruminating and feeding behaviors will be more variable when compared with visual observations.

\section{MATERIALS AND METHODS}

This study was conducted at the University of Kentucky Coldstream Dairy Research Farm in Lexington under Institutional Animal Care and Use Committee protocol number 2014-1309. The lactating herd (80 cows) was housed in 2 equal groups separated by a shared, raised feedbunk with a conveyer feed delivery system. A TMR ration containing corn silage, alfalfa silage, whole cottonseed, and grain mix was delivered twice daily, at 0530 and $1330 \mathrm{~h}$. Cows were provided with unrestricted access to freestalls. One group of cows was provided sawdust-covered rubber-filled mattresses (PastureMat, Promat, Ontario, Canada). The other group of cows was provided sawdust-covered Dual Chamber Cow Waterbeds (Advanced Comfort Technology Inc., Reedburg, WI). Grass-seeded exercise lot access was permitted for $1 \mathrm{~h}$ per day at $1000 \mathrm{~h}$, weather permitting. All other surfaces (freestall area, feedbunk alley, holding pen, and alleys) contained grooved concrete. Milking occurred twice daily at 0430 and $1530 \mathrm{~h}$. All cattle within the herd were fitted with the following technologies before or at calving, as per standard herd protocol (further information presented in Table 1): AfiAct Pedometer Plus, CowManager SensOor, CowAlert IceQube, Smartbow, and Track A Cow.

The number of cattle needed for our study was determined using the methods of Friedman (1982). All power tests were calculated to obtain a power $(1-\beta)$ of 0.90 and a type I error probability $(\alpha)$ of 0.05 (2-tailed). Effect size was determined by previous studies (equal to correlation coefficients; Bikker et al., 2014) and was 0.93 for rumination time (Bikker et al., 2014), 0.88 for feeding time (Bikker et al., 2014) and 0.90 for lying time (Ledgerwood et al., 2010). A minimum of 24 cows was needed to meet statistical power requirements and this number was doubled to account for potential instances of missing data $(\mathrm{n}=48)$. Eligible cattle were randomly selected and balanced for herd group and parity. No cattle within 21 DIM were included in our study to remove potential effects from the transition period. The 
BORCHERS ET AL.

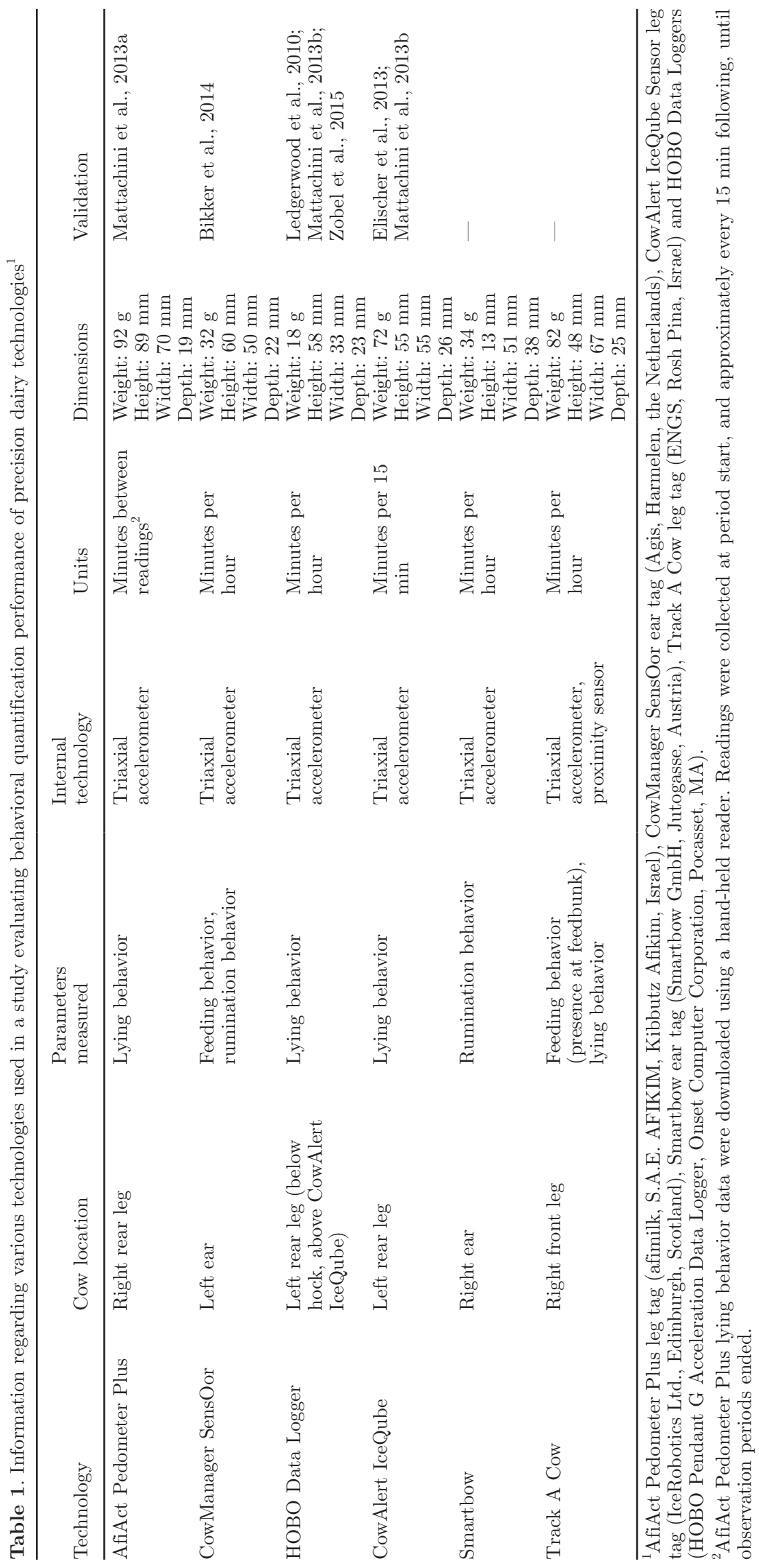


final sample population consisted of 24 primiparous and 24 multiparous $(\mathrm{n}=48)$ Holstein dairy cows at a mean $223.4 \pm 117.8$ DIM, producing a mean of $29.22 \pm 8.20$ $\mathrm{kg}$ of milk per day. Additionally, HOBO Data Loggers were placed in watertight bags, wrapped in self-adhesive wrap, and attached to each cow's lower left rear leg (15 $\mathrm{cm}$ above the CowAlert IceQube), approximately 12 $\mathrm{h}$ before observation began. The HOBO Data Loggers recorded lying behavior using a triaxial accelerometer to collect relative position every minute. Unadjusted HOBO Data Logger data were collected and prepared for analysis using established data conversion methods provided by the University of British Columbia (UBC, 2013). The HOBO lying behavior data were used as a basis for comparison because previous studies have established the effectiveness of HOBO Data Loggers in 1-min periods (Ito et al., 2009).

\section{Behavior Classification}

Parameters recorded using technologies were compared with direct visual observation, which took place over 8 d. Each of the 48 enrolled cows was assigned 1 $\mathrm{d}$, resulting in 6 cows being observed per day. Each cow was observed for a total of $4 \mathrm{~h}$, split into two 2-h observation periods. Observation occurred following morning and evening milking as cows exited the milking parlor. Each cow was observed for 1 morning and 1 evening period. Observers consisted of 42 undergraduate and graduate students from the University of Kentucky. Six observers were assigned to each period, 1 per cow. Fourteen observers contributed only 1 observation period and the remaining 28 observers contributed multiple observation periods.

Data-recording sheets and event classification instructions were sent to each observer several days before their period. Upon arrival at the dairy and before the beginning of each observational period, all observers were instructed on proper behavioral classification and recording techniques by a mutual instructor (same throughout the study). Example videos of each behavior were shown to observers exemplifying feeding, rumination, and lying or standing events. Observers were instructed on proper recording procedures for these behaviors using these same videos for reference. For the safety of observers and cattle, observers were instructed to disrupt cattle as little as possible. If cattle had not performed an eating or lying event toward the end of observation periods, behaviors were encouraged by the researcher who performed the training. For example, cows lying for the entire observation period were encouraged to stand, similar to methods used by Bonk et al. (2013) to generate standing bouts. Cattle were also gently guided to feeding areas if eating events had not yet occurred. Decisions to encourage cow behaviors were made by the lead researcher and were not left to observer discretion.

At the commencement and cessation of each observed behavior, observers recorded the hour, minute, and second of the day using multifunction, radio frequency synchronized, atomic watches (Casio America Inc., Dover, NJ). Behavioral commencement and cessation times were used to calculate the duration behaviors were performed for each observational period.

A cow was considered to be eating if actively chewing and standing near the feedbunk. If chewing stopped for longer than $5 \mathrm{~s}$, cattle were recorded as having stopped eating. Rumination was quantified similar to Schirmann et al. (2009), where rumination was defined as the point in time of bolus regurgitation. Observers recorded events where regurgitated boluses reached the esophagus, entered the mouth, and were subsequently followed by the initiation of rhythmic chewing. Rumination events ended when rhythmic chewing ceased and the bolus was swallowed. Similar to the methods of Ledgerwood et al. (2010), transition between standing and lying positions defined lying behavior. Cattle were considered lying when the flank of the animal came in contact with a surface while transitioning from a standing position. A cow was classified as standing when all 4 limbs were fully extended and perpendicular to the ground while transitioning from a lying position.

\section{Interobserver Variability}

A method using 4 observers established interobserver variability. The lead author, who instructed all observers before each observation period, served as observer 1 . For both a morning and evening observation period $(4 \mathrm{~h}$ total), 4 observers recorded behavioral data from $1 \mathrm{cow}$ in the same methods as previously described. Observers were instructed not to look at the others' recordings, or share data. Observations were recorded using the aforementioned techniques and tested for agreement at each second during the observation period. The CORR procedure of SAS version 9.3 (SAS Institute, Cary, NC) generated Pearson correlation coefficients among all observers, and all correlations throughout the study were classified according to criteria used by Bikker et al. (2014): negligible, 0.00 to 0.30 ; slight, 0.31 to 0.50 ; minor, 0.51 to 0.70 ; moderate, 0.71 to 0.90 ; and strong, 0.91 to 1.00 . A chi-squared test was performed using $\mathrm{R}$ version 3.1.0 ( $\mathrm{R}$ Foundation for Statistical Computing, Vienna, Austria) to evaluate observer agreement on behaviors performed at each second. The FREQ procedure of SAS was also used to calculate a Cohen's kappa coefficient $(\kappa)$ between individual observers at each second. All $\kappa$-values were classified according to 
criteria of Landis and Koch (1977), with poor, <0.00; slight, 0.00 to 0.20 ; fair, 0.21 to 0.40 ; moderate, 0.41 to 0.60 ; substantial, 0.61 to 0.80 ; and near perfect, 0.81 to 1.00 .

\section{Data Preparation and Statistical Analysis}

The difference between observation and technology data was calculated and differences below the 5th and above the 95th percentile were identified as outliers and removed from further analysis. Technology and visual observation data were averaged by cow to provide 1 observation of each per subject (cow). These averages were used to establish agreement between technologies and visual observations (Bland and Altman, 1995a,b), using the CORR procedure of SAS to generate Pearson correlation coefficients. Agreement was further compared using bias correction factors $\left(\mathrm{C}_{\mathrm{b}}\right)$, concordance correlation coefficients (CCC), location shift values $(\mathrm{V})$, and scale shift values $(\mu)$ using the "epiR" package in $\mathrm{R}$ version 3.1.0 to test individual technologies against visual observations and those generated by the HOBO Data Logger for lying behaviors.

\section{RESULTS AND DISCUSSION}

\section{Interobserver Variability}

Correlations and $\kappa$-coefficients among observers can be found in Table 2a. Strong correlations were established among observers for feeding behavior $(\mathrm{r}>0.96$ across observers, $P<0.01)$ and lying time $(\mathrm{r}>0.99$ across all observers, $P<0.01)$. Rumination time was most variable among observers $(\mathrm{r}>0.88$ across all observers, $P<0.01$ ). All $\kappa$-values indicated agreement with Pearson correlation coefficients for feeding behavior ( $\kappa>0.96$ across observers) and lying time ( $\kappa$ $>0.99$ across all observers). Rumination time was also most variable among observers for $\kappa$-values $(\kappa>0.88$ across all observers). Table $2 \mathrm{~b}$ displays results of a chisquared analysis on interobserver data. No significant differences were found among observers for rumination or lying behaviors $(P>0.05)$, but significant differences occurred for feeding time. Although one instance proved significant for this test, these differences were numerically small. The proportion of time these behaviors were performed for the observation period was 0.26 for observers 1 to 3 and 0.25 for observer 4. Agreement among observers was evaluated for each second over a 4-h period (14,400 records) and the number of observations may have influenced significance for this particular test. Because these numbers were numerically small and the other tests indicated moderate to strong correlation and near perfect $\kappa$-values, visual observations were regarded as reliable. Future studies comparing technologies to visual observations may benefit from using fewer observers.

\section{Outlier Removal}

The outlier removal method resulted in 2 cows being removed entirely from each technology monitoring hourly behavioral data $(\mathrm{n}=46)$. No cows were removed from the CowAlert IceQube or AfiAct Pedometer Plus systems due to the data periods provided by those technologies. The Track A Cow system had an additional 2 cows removed from the lying behavior analysis $(\mathrm{n}=44)$ and feeding behavior analyses due to data transmission errors for that parameter.

\section{Feeding Behavior}

Technologies recorded feeding behavior in minutes per 1-h time block and were evaluated against visual observations over the same period; results are shown in Table 3. Technology data correlated well with visual observation data for the CowManager SensOor ( $\mathrm{r}=$ $0.88, P<0.01 ; \mathrm{CCC}=0.82)$ and the Track A Cow $(\mathrm{r}$ $=0.93, P<0.01 ; \mathrm{CCC}=0.79)$ systems. Bikker et al. (2014) previously evaluated the CowManager SensOor and showed similar results $(\mathrm{r}=0.88, P<0.01$; CCC $=0.75)$.

To our knowledge, the current study is the first to validate the Track A Cow system. Systems such as the CowManager SensOor monitor feeding time using head movements, whereas the Track A Cow more accurately describes feeding behavior or time around the feed bunk. A similar system recording cow proximity to the feedbunk was previously shown to be highly correlated with observed feeding behavior $\left(\mathrm{R}^{2}=0.98 ; P<0.01\right.$; DeVries et al., 2003). The system used in DeVries et al. (2003) recorded feeding events when cattle placed their heads under feed rails and over feed. A system monitoring a cow's presence at the feedbunk was also evaluated by Chapinal et al. (2007) and strongly correlated with visual observation $\left(\mathrm{R}^{2}=1.00 ; P<0.01\right)$. The current study indicated the CowManager SensOor and Track A Cow systems to underperform these other technologies; however, these other technologies are primarily used as research tools. The 2 technologies evaluated in our study were marketed specifically for use on commercial dairy farmers.

\section{Rumination}

Technologies recorded rumination in minutes per 1-h time block and were evaluated against visual observations over the same period; results are shown in Table 
Table 2. Results from a technology evaluation study indicating agreement between 4 observers for visual recordings of feeding behavior, lying time, and rumination time on the same cow using Pearson correlation coefficient and $\kappa$ values \pm ASE, and chi-squared values ${ }^{1,2}$

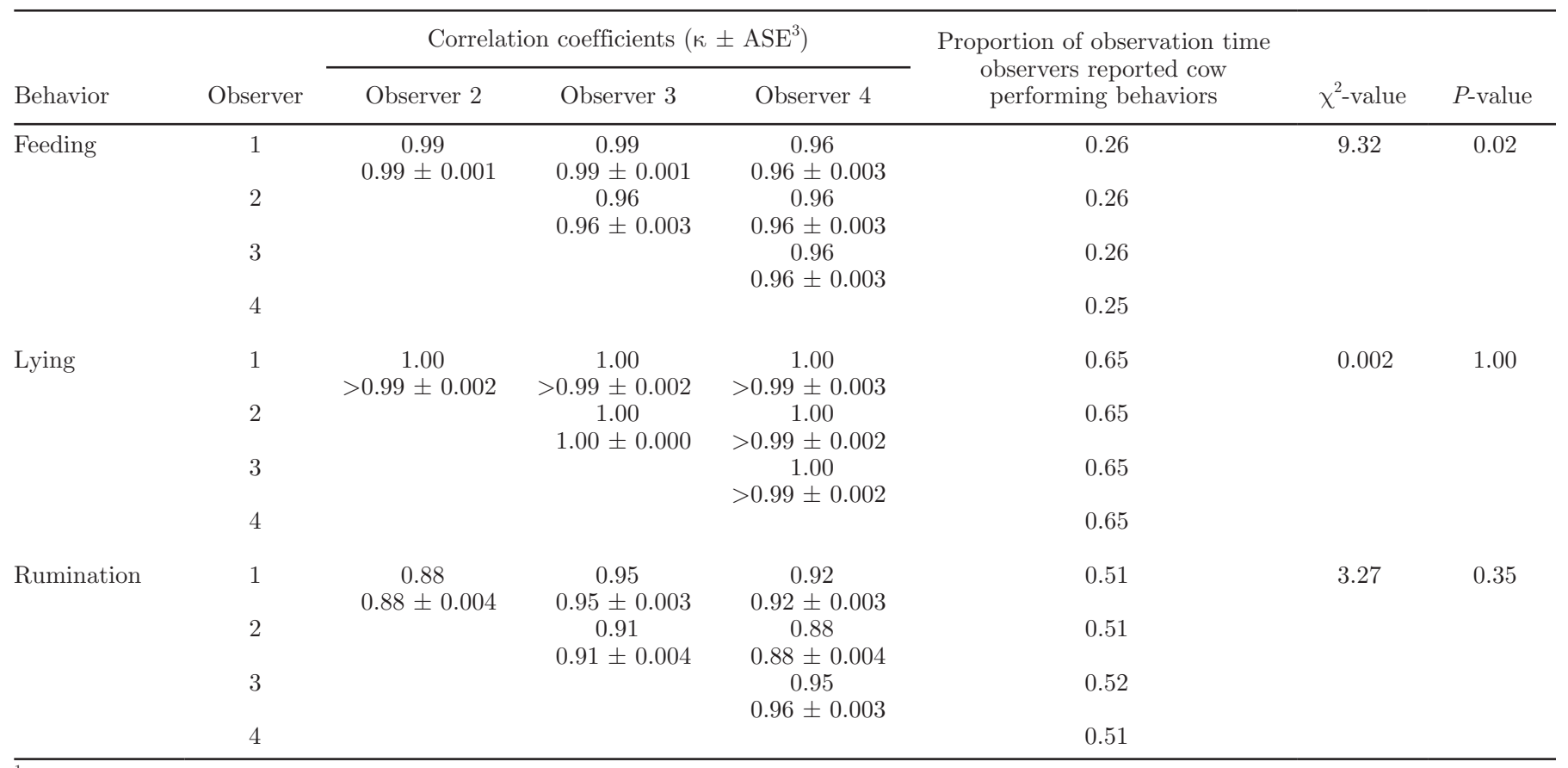

${ }^{1}$ All Pearson correlation coefficients were evaluated for the probability of observing results under the null hypothesis of observations being unrelated.

${ }^{2}$ All Pearson correlation coefficient values were significance at $P<0.01$

${ }^{3}$ Asymptomatic standard error.

3. CowManager SensOor data were more weakly correlated with visual observations $(\mathrm{r}=0.69, P<0.01$; CCC $=0.59)$ than the Smartbow system $(\mathrm{r}=0.97, P<0.01$; $\mathrm{CCC}=0.96)$. In a previous evaluation of the CowManager SensOor, rumination was strongly correlated with visual observation $(\mathrm{r}=0.93, P<0.01 ; \mathrm{CCC}=0.93$; Bikker et al., 2014). In the current study, rumination was the most subjective for observers to evaluate and showed slight variation among observers $(\mathrm{r}=0.89, P$ $<0.01$; interobserver variability). The evaluation of rumination behaviors has traditionally been completed in tiestalls, small pens, or a similar controlled setting
(Schirmann et al., 2009; Bikker et al., 2014). The current study allowed cattle to express behaviors as they would in a general herd, potentially causing observers to miss or misidentify rumination events. Misidentified visual observation events could skew the mean values used in our study, generating weaker correlations.

The technologies evaluated in our study use accelerometers to detect rumination events. Few technologies using accelerometers for rumination behavior classification have been evaluated. Previous studies have monitored jaw movements (Zehner et al., 2012) or eructation sounds (Schirmann et al., 2009) to quantify rumination

Table 3. Results from a technology evaluation study with Pearson correlation coefficients $(\mathrm{r})$, bias correction factor $\left(\mathrm{C}_{\mathrm{b}}\right)$, concordance correlation coefficient (CCC), location shift (V), and scale shift $(\mu)$ for hourly summed values indicating agreement between visual observations and feeding or rumination data collected by various precision monitoring technologies ${ }^{1,2}$

\begin{tabular}{llcrrrrr}
\hline Behavior & Technology & $\begin{array}{c}\text { Number } \\
\text { of cows }\end{array}$ & $\mathrm{r}$ & $\mathrm{C}_{\mathrm{b}}$ & $\mathrm{CCC}$ & $\mathrm{V}$ & \multicolumn{1}{c}{} \\
\hline Feeding & CowManager SensOr & 46 & 0.88 & 0.94 & 0.82 & 0.32 \\
& Track A Cow & 41 & 0.93 & 0.85 & 0.79 & 0.47 \\
Rumination & CowManager SensOor & 46 & 0.69 & 0.85 & 0.59 & -0.58 & 1.41 \\
& Smartbow & 46 & 0.97 & 0.99 & 0.96 & 0.14 & 1.06 \\
\hline
\end{tabular}

${ }^{1}$ CowManager SensOor ear tag (Agis, Harmelen, the Netherlands), Smartbow ear tag (Smartbow GmbH, Jutogasse, Austria), and the Track A Cow leg tag (ENGS, Rosh Pina, Israel).

${ }^{2}$ All Pearson correlation coefficient values were significance at $P<0.01$. 
behavior. Results of the current study indicate some accelerometers may underperform alternative methods, but more research is needed.

\section{Lying Behavior}

The AfiAct Pedometer Plus, CowAlert IceQube, and Track A Cow monitors were evaluated against visual observations and HOBO Data Loggers; results are shown in Table 4 . The CowAlert IceQube correlated strongly with visual observations at $\mathrm{r}>0.99(P$ $<0.01)$ and CCC $>0.99$. Elischer et al. (2013) evaluated the IceQube's ability to measure lying behavior using a Pearson correlation coefficient and found similar correlation to the current study $(\mathrm{r}=0.97 ; P<$ 0.01). The IceTag was previously evaluated for accuracy (McGowan et al., 2007; Mattachini et al., 2013b), but the IceTag is intended for use as a research tool whereas the CowAlert IceQube used in our study was the commercially marketed version. Mattachini et al. (2013b) found the IceTag to perform similarly to video observation with a sensitivity of (mean \pm SD) $1.00 \pm$ $<0.01$, and a specificity of $1.00 \pm<0.01$. Mattachini et al. (2013b) found observations from the HOBO Data Logger and IceTag to correspond with a sensitivity of $0.99 \pm<0.01$ and a specificity of $0.99 \pm<0.01$. In the current study, HOBO Data Logger performance was also compared with visual observations on a 15-min basis and a moderate correlation was observed $(\mathrm{r}=$ $0.87, P<0.01 ; \mathrm{CCC}=0.87)$.

The Track A Cow system was strongly correlated with visual observation $(\mathrm{r}>0.99, P<0.01 ; \mathrm{CCC}$ $>0.99$ ). The Track A Cow is placed on the front right leg, and previous studies have shown the front legs to be less accurate in monitoring lying behavior (Müller and Schrader, 2003). Hourly summed data from the HOBO Data Loggers were strongly correlated with the Track A Cow $(\mathrm{r}=0.92, P<0.01 ; \mathrm{CCC}=0.92)$. These results indicate lying behavior monitored on front and hind limbs to be similar.

AfiAct Pedometer Plus and visual observations were highly correlated $(\mathrm{r}>0.99, P<0.01$; CCC $>0.99)$, whereas AfiAct Pedometer Plus and HOBO Data Logger observations were less highly correlated than any other technology $(\mathrm{r}=0.83, P<0.01 ; \mathrm{CCC}=0.81)$. The method (hand-held reader) used to collect AfiAct Pedometer Plus lying behavior data may have influenced results. The AfiAct Pedometer Plus used software that delayed data generation to account for potentially erroneous readings. Many technologies must remain in a lying or standing position for a predetermined and proprietary period of time to register a lying or standing event (Ledgerwood et al., 2010; Mattachini et al., 2013a). Because of this, the tag tended to overestimate or underestimate lying time in comparison to visual observations and HOBO Data Logger readings. If the hand-held reader collected lying behavior before data delays were complete, time lying or standing for those readings would be passed to subsequent time blocks, misrepresenting data. With continuously recorded data, delayed data would have a lesser effect on results. Future studies will need to establish the effectiveness of the AfiAct Pedometer Plus in automatically collecting lying behavioral data at regular intervals. Automatic data collection was available for this technology at the time of our study, but was not yet available at the study farm.

Table 4. Results from a technology evaluation study with Pearson correlation coefficients $(\mathrm{r})$, bias correction factor $\left(\mathrm{C}_{\mathrm{b}}\right)$, concordance correlation coefficient (CCC), location shift $(\mathrm{V})$, and scale shift $(\mu)$ values indicating agreement between technologies, visual observations, and the HOBO Data Logger in recording lying time in Holstein dairy cattle ${ }^{1,2}$

\begin{tabular}{|c|c|c|c|c|c|c|}
\hline Behavioral monitoring methods compared ${ }^{3}$ & $\begin{array}{l}\text { Number } \\
\text { of cows }\end{array}$ & $\mathrm{r}$ & $\mathrm{C}_{\mathrm{b}}$ & CCC & $\mathrm{V}$ & $\mu$ \\
\hline CowAlert IceQube with visual observation & 48 & $>0.99$ & $>0.99$ & $>0.99$ & $<0.00$ & 1.00 \\
\hline CowAlert IceQube with HOBO Data Logger & - & 0.87 & 0.99 & 0.87 & 0.11 & 1.13 \\
\hline Track A Cow with visual observation & 44 & $>0.99$ & 0.99 & 0.99 & -0.11 & 0.97 \\
\hline Track A Cow with HOBO Data Logger & - & 0.92 & $>0.99$ & 0.92 & $<0.00$ & 1.03 \\
\hline HOBO Data Logger with visual observation & - & 0.93 & 0.99 & 0.92 & 0.10 & 1.07 \\
\hline HOBO Data Logger with visual observation & - & 0.83 & 0.98 & 0.81 & 0.14 & 1.16 \\
\hline
\end{tabular}

${ }^{1}$ AfiAct Pedometer Plus leg tag (Afimilk, S.A.E. AFIKIM, Kibbutz Afikim, Israel), CowAlert IceQube Sensor leg tag (IceRobotics Ltd., Edinburgh, Scotland), Track A Cow leg tag (ENGS, Rosh Pina, Israel), and HOBO Data Loggers (HOBO Pendant G Acceleration Data Logger, Onset Computer Corporation, Pocasset, MA).

${ }^{2}$ All Pearson correlation coefficient values were significance at $P<0.01$.

${ }^{3}$ Observational and $\mathrm{HOBO}$ data were summed in $15 \mathrm{~min}$, hourly, and variable time blocks to match technology data summation times. Comparisons were performed between HOBO Data Loggers and observed data to match technology time intervals.

${ }^{4}$ Data for the AfiAct Pedometer Plus system were collected using a hand-held reader approximately once every 15 min. 
The HOBO Data Logger correlations to visual observations and technology data were nearly identical on a 15-min basis (HOBO Data Loggers to visual: $\mathrm{r}=0.88$, $P<0.01, \mathrm{CCC}=0.87$; HOBO Data Loggers to CowAlert IceQube: $\mathrm{r}=0.87, P<0.01, \mathrm{CCC}=0.87)$, hourly basis (HOBO Data Loggers to visual: $\mathrm{r}=0.93, P<$ $0.01, \mathrm{CCC}=0.92$; HOBO Data Loggers to Track A Cow: $\mathrm{r}=0.92, P<0.01, \mathrm{CCC}=0.92)$, and the variable basis used for the AfiAct Pedometer Plus (HOBO Data Loggers to visual: $\mathrm{r}=0.83, P<0.01, \mathrm{CCC}=0.81$; HOBO Data Loggers to technology: $\mathrm{r}=0.92, P<0.01$, $\mathrm{CCC}=0.81$ ). The HOBO Data Loggers have previously been shown to accurately describe lying behavior in dairy cattle (Ledgerwood et al., 2010; Mattachini et al., 2013a,b), dairy calves (Bonk et al., 2013), and dairy goats (Zobel et al., 2015). To corroborate the current study, visual observations have been shown to be more similar to the AfiAct Pedometer Plus and IceTag than to the HOBO Data Logger (Mattachini et al., 2013a,b), which could be due to several reasons. The HOBO Data Loggers are research tools and have differing sampling times. In this experiment, the HOBO Data Loggers sampled the device's 3-dimensional position every minute. Data analysis techniques assumed this position to remain constant for each minute. This could lead to variation in the number of minutes spent lying in comparison to technologies sampling more frequently.

\section{Further Discussion}

The technologies used in the current study were able to accurately quantify feeding, rumination, and lying behaviors. Commercially marketed technologies showed only slight differences in correlation with visual observations. The HOBO Data Loggers (in lying time evaluation only) were less strongly correlated with visual observations than any commercially marketed technologies. This indicates commercially marketed lying behavior monitors to be more effective than HOBO Data Loggers in quantifying lying behavior. Based on these results, many commercially marketed technologies may be more useful in research settings than the HOBO Data Logger. An additional reason for this finding may be the data conversion methods used for the HOBO Data Loggers. Adjusting the method by which raw data were converted to lying data may yield a greater correlation between the HOBO Data Loggers and visual observations or technology data. Previous methods have evaluated the HOBO Data Loggers' performance over different sampling time frequencies (Ito et al., 2009; Ledgerwood et al., 2010; Mattachini et al., 2013b) and adjustments in sampling frequency may also increase technology performance.
Comparing all data using the same time intervals may provide more accurate technology comparisons, but this was not possible in the current study. Technologies collected data with different frequencies between sampling. Summation of technology and observation data into hour blocks would have allowed for all but the AfiAct Pedometer Plus (because of variable time blocks) to be compared. This was not performed because technology manufacturers describe technology-recorded behaviors in different time blocks. Manipulating technology data into common time blocks would not accurately represent the data used to perform the respective functions of the technologies (e.g., health and estrus detection). Changing the format by which data are presented could misrepresent data used in algorithms, creating biased comparisons. Future research obtaining technology data in common time units directly from manufacturers would allow for a more accurate comparison of technology performance.

\section{CONCLUSIONS}

To our knowledge, this is the first precision dairymonitoring technology validation study evaluating multiple parameters and technologies attached to the same cows. This is also the first study to validate parameters measured by the Track A Cow and Smartbow systems. Commercially marketed technologies recording feeding behavior, rumination, and lying behavior performed well when compared with visual observation. Rumination behavior was most variable from visual observation. Similar performance to visual observations and other validated technologies indicates dairy cattle behavioral monitors to provide accurate information for use in dairy cow management.

\section{ACKNOWLEDGMENTS}

The authors thank S.A.E. AFIKIM (Afikim, Israel), Agis (Harmelen, the Netherlands), IceRobotics Ltd. (Edinburgh, UK), Smartbow GmbH (Jutogasse, Austria), and ENGS (Rosh Pina, Israel) for allowing us to use their technologies in the study. The authors also thank the Applied Statistics Lab at the University of Kentucky, specifically Sarah Janse and Constance Wood, for providing statistical support. Additionally, we thank the University of Kentucky Coldstream Dairy (Lexington) staff for their help with this study and Alexis Thompson, Alison DiGennaro, Amanda Stone, Barbara Wadsworth, Barbara Wolfger, Bettie Kawonga, Caitlin Quinn, Cameron Earlywine, Cheyenne Vercosa, Denise Ray, Derek Nolan, Emily Lunsford, Emory Thomas, Gustavo Mazon, Israel Mullins, Jamie Leistner, Jef- 
frey Bewley, Jeffrey Hamilton, Jenna Mangiarelli, Jenna Sturm, Jordan Martinez, Karmella Dolecheck, Kathryn Tucker, Katie Kelly, Lauren Mayo, Elizabeth Eckelkamp, Luke Arthur, Maegan Weatherly, Matthew Jeffiers, Mauricio Xavier da Silva Oliveira, Meagan Woodard-Berwanger, Megan Hardy, Mickayla Myers, Naara Macedo, Nayara Magalhaes, I Ching (Nicky) Tsai, Savannah Meade, Sophie Dorsch, Stefanie Rogers, Victoria Casso, Vinicius Goncalves, Greg Wheeler, and Ingrid Brito who so graciously volunteered to observe the cows in this study. The authors also thank Gosia Zobel and the University of British Columbia (Vancouver, Canada) for assisting with the HOBO Data Logger lying behavior data conversion.

\section{REFERENCES}

Beauchemin, K. A., S. Zelin, D. Genner, and J. G. Buchanan-Smith 1989. An automatic system for quantification of eating and ruminating activities of dairy cattle housed in stalls. J. Dairy Sci. 72:2746-2759.

Bikker, J. P., H. van Laar, P. Rump, J. Doorenbos, K. van Meurs, G. M. Griffioen, and J. Dijkstra. 2014. Technical note: Evaluation of an ear-attached movement sensor to record cow feeding behavior and activity. J. Dairy Sci. 97:2974-2979.

Bland, J. M., and D. G. Altman. 1995a. Calculating correlation coefficients with repeated observations: Part 1 - Correlation within subjects. BMJ 310:446.

Bland, J. M., and D. G. Altman. 1995b. Calculating correlation coefficients with repeated observations: Part 2-correlation between subjects. BMJ 310:633.

Bonk, S., O. Burfeind, V. S. Suthar, and W. Heuwieser. 2013. Technical note: Evaluation of data loggers for measuring lying behavior in dairy calves. J. Dairy Sci. 96:3265-3271.

Chapinal, N., D. M. Veira, D. M. Weary, and M. A. G. von Keyserlingk. 2007. Technical note: Validation of a system for monitoring individual feeding and drinking behavior and intake in grouphoused cattle. J. Dairy Sci. 90:5732-5736.

DeVries, T. J., M. A. von Keyserlingk, D. M. Weary, and K. A. Beauchemin. 2003. Technical note: Validation of a system for monitoring feeding behavior of dairy cows. J. Dairy Sci. 86:3571-3574.

Elischer, M. F., M. E. Arceo, E. L. Karcher, and J. M. Siegford. 2013. Validating the accuracy of activity and rumination monitor data from dairy cows housed in a pasture-based automatic milking system. J. Dairy Sci. 96:6412-6422.

Friedman, H. 1982. Simplified determinations of statistical power, magnitude of effect and research sample sizes. Educ. Psychol. Meas. 42:521-526.

Haley, D. B., J. Rushen, and A. M. de Passille. 2000. Behavioural indicators of cow comfort: Activity and resting behaviour of dairy cows in two types of housing. Can. J. Anim. Sci. 80:257-263.

Ito, K., D. M. Weary, and M. A. von Keyserlingk. 2009. Lying behavior: Assessing within- and between-herd variation in free-stallhoused dairy cows. J. Dairy Sci. 92:4412-4420.

Landis, J. R., and G. G. Koch. 1977. An application of hierarchical kappa-type statistics in the assessment of majority agreement among multiple observers. Biometrics 33:363-374.
Ledgerwood, D. N., C. Winckler, and C. B. Tucker. 2010. Evaluation of data loggers, sampling intervals, and editing techniques for measuring the lying behavior of dairy cattle. J. Dairy Sci. 93:5129-5139.

Kononoff, P. J., H. A. Lehman, and A. J. Heinrichs. 2002. Technical note - A comparison of methods used to measure eating and ruminating activity in confined dairy cattle. J. Dairy Sci. 85:1801-1803.

Mattachini, G., A. Antler, E. Riva, A. Arbel, and G. Provolo. 2013a. Automated measurement of lying behavior for monitoring the comfort and welfare of lactating dairy cows. Livest. Sci. 158:145-150.

Mattachini, G., E. Riva, C. Bisaglia, J. Pompe, and G. Provolo. 2013b. Methodology for quantifying the behavioral activity of dairy cows in freestall barns. J. Anim. Sci. 91:4899-4907.

McGowan, J., C. Burke, and J. Jago. 2007. Validation of a technology for objectively measuring behaviour in dairy cows and its application for oestrous detection. Page 136 in Proceedings-New Zealand Society of Animal Production. New Zealand Society of Animal Production, Wanaka, New Zealand.

Müller, R., and L. Schrader. 2003. A new method to measure behavioural activity levels in dairy cows. Appl. Anim. Behav. Sci. 83:247-258.

O'Driscoll, K., L. Boyle, and A. Hanlon. 2008. A brief note on the validation of a system for recording lying behaviour in dairy cows. Appl. Anim. Behav. Sci. 111:195-200.

Proudfoot, K. L., M. B. Jensen, D. M. Weary, and M. A. G. von Keyserlingk. 2014. Dairy cows seek isolation at calving and when ill. J. Dairy Sci. 97:2731-2739.

Rutten, C. J., A. G. Velthuis, W. Steeneveld, and H. Hogeveen. 2013. Invited review: Sensors to support health management on dairy farms. J. Dairy Sci. 96:1928-1952.

Schirmann, K., M. A. von Keyserlingk, D. M. Weary, D. M. Veira, and W. Heuwieser. 2009. Technical note: Validation of a system for monitoring rumination in dairy cows. J. Dairy Sci. 92:6052-6055.

Senger, P. L. 1994. The estrus detection problem: New concepts, technologies, and possibilities. J. Dairy Sci. 77:2745-2753.

UBC. 2013. UBC Animal Welfare Program: SOP-HOBO Data Loggers. University of British Columbia, Vancouver, Canada. Accessed Nov. 1, 2013. http://awp.landfood.ubc.ca/files/2013/11/ SOP-HOBO-Datalogger-november-2013.pdf.

Wathes, C. M., H. H. Kristensen, J. M. Aerts, and D. Berckmans. 2008. Is precision livestock farming an engineer's daydream or nightmare, an animal's friend or foe, and a farmer's panacea or pitfall? Comput. Electron. Agric. 64:2-10.

Weary, D. M., J. Huzzey, and M. von Keyserlingk. 2009. Board-invited review: Using behavior to predict and identify ill health in animals. J. Anim. Sci. 87:770-777.

Zehner, N., J. Niederhauser, F. Nydegger, A. Grothmann, M. Keller, M. Hoch, A. Haeussermann, and M. Schick. 2012. Validation of a new health monitoring system (rumiwatch) for combined automatic measurement of rumination, feed intake, water intake and locomotion in dairy cows. Pages C-0438 in Proc. Infomation Technology, Automation and Precision Farming. International Conference of Agricultural Engineering-CIGR-AgEng 2012: Agriculture and Engineering for a Healthier Life, Valencia, Spain, 8-12 July 2012. CIGR-EurAgEng, Cranfield, UK.

Zobel, G., D. M. Weary, K. Leslie, N. Chapinal, and M. A. von Keyserlingk. 2015. Technical note: Validation of data loggers for recording lying behavior in dairy goats. J. Dairy Sci. 98:1082-1089. 